\title{
Magnetic activity in the young solar analog $A B$ Dor
}

\section{Active longitudes and cycles from long-term photometry ${ }^{\star}$}

\author{
S. P. Järvinen ${ }^{1,2,3}$, S. V. Berdyugina ${ }^{3,4}$, I. Tuominen ${ }^{3}$, G. Cutispoto ${ }^{5}$, and M. Bos ${ }^{6}$ \\ 1 Astrophysikalisches Institut Potsdam, An der Sternwarte 16, 14482 Potsdam, Germany \\ 2 Nordic Optical Telescope, Apartado 474, 38700 Santa Cruz de La Palma, Spain \\ 3 Astronomy Division, PO Box 3000, 90014 University of Oulu, Finland \\ e-mail: silva.jarvinen@iki.fi \\ 4 Institute of Astronomy, ETH Zentrum, 8092 Zürich, Switzerland \\ 5 INAF - Catania Astrophysical Observatory, v. S. Sofia 78, 95123 Catania, Italy \\ ${ }^{6}$ Molehill Astronomical Observatory, 54 McDowell Crescent, Glenfield, North Shore City, New Zealand
}

Received 13 September 2004 / Accepted 5 November 2004

\begin{abstract}
We analyse photometric observations of the young active dwarf AB Dor, spanning more than 20 years. Similar to the young solar analog LQ Hya, AB Dor shows long-lived, nonaxisymmetric spot distribution - active longitudes in opposite hemispheres. The active longitudes migrate nonlinearly in the fixed reference frame, because of the differential rotation and changes of the mean spot latitudes. At least two activity cycles are found in the data. One cycle originates from repeating switches of the activity between the two active longitudes in about (2-3)-year intervals. This results in the flip-flop cycle of about 5.5 years, which includes two consecutive switches. The 5.5-yr cycle also modulates variations of the minimum stellar brightness and the peak-to-peak amplitude, that suggests a periodic redistribution of the spot area between the opposite longitudes and supports the reality of the flip-flop cycle. The other cycle is clearly seen in variations of the mean and maximum stellar brightness on the time-scale of 20 years and is reminiscent of the 11-year sunspot cycle.
\end{abstract}

Key words. stars: activity - stars: starspots

\section{Introduction}

$\mathrm{AB}$ Dor (HD 36705) is an active rapidly rotating K0 dwarf in the southern sky. It rotates about 50 times faster than the Sun $(P=0.51479)$ and exhibits very strong magnetic activity. The distance to the star of $14.9 \mathrm{pc}$ measured by HIPPARCOS combined with the brightest state so far observed $V=6.75$ (Amado et al. 2001) leads to the absolute magnitude $M_{V}=5.89$. Rucinski (1983) and Innis et al. (1986) concluded AB Dor to be a pre-main-sequence star, but this was questioned by Micela et al. (1997) on the basis of the distance. More likely AB Dor is a zero-age main-sequence star. The study by Innis et al. (1986) indicated that according to the Galactic space motions, $U, V, W$, $\mathrm{AB}$ Dor is a member of the Pleiades moving group, which would make its age between $\sim 70-100 \mathrm{Myr}$. However, a recent study by Zuckerman et al. (2004) of Galactic space motions indicates that the star could belong to an even younger group of stars of $\sim 50 \mathrm{Myr}$ old called the AB Dor moving group. The young age of the star is confirmed by the high lithium abundance (e.g. Rucinski 1982; Hussain et al. 1997).

* Tables 2 and 3 are only available in electronic form at the CDS via anonymous ftp to cdsarc.u-strasbg.fr $(130.79 .128 .5)$ or via http://cdsweb.u-strasbg.fr/cgi-bin/qcat?]/A+A/432/657
There are many indications of magnetic activity in AB Dor. Its optical brightness is highly variable because of rapidly evolving cool spots on the stellar surface. Long-term photometry of the star indicates that the mean brightness of the star has decreased since its first observations in 1978 until around 1988, when it reached its minimum, and increased afterwards (Amado et al. 2001), until, probably somewhere between 2000 and 2004, it reached its maximum. This may be evidence for a solar-type cycle with varying spot coverage which influences the total brightness of the star. The latter was found to be on a time scale of between 18 and 21 years with a possible additional periodicity of about 5.3 years (Amado et al. 2001).

The magnetic activity of $\mathrm{AB}$ Dor is also evident in strong $\mathrm{Ca}$ II and $\mathrm{H} \alpha$ emission, that suggests the presence of an active chromosphere (Bidelman \& MacConnell 1973; Vilhu et al. 1987). The stellar corona produces strong and variable radio and X-ray radiation (Slee et al. 1986; Pakull 1981). The radio emission of $\mathrm{AB}$ Dor varies periodically over many rotations, suggesting that the magnetic field structure(s) containing each radio source must persist at the same stellar longitude(s) for long time intervals. Moreover, according to Lim et al. (1994), radio emission peaks seemed to be centred at or near rotation phases of spot concentrations observed in the 
optical wavebands. This suggests that the star may exhibit longlived active longitudes in the photosphere, chromosphere and corona. Long rise-times of X-ray flares suggest that they occur in loop structures with lengths of several stellar radii (Collier Cameron et al. 1988).

AB Dor has been extensively studied with Doppler imaging techniques (Kürster et al. 1994; Collier Cameron \& Unruh 1994; Collier Cameron 1995; Donati \& Collier Cameron 1997; Donati et al. 1999, 2003; Hussain et al. 2002). The Doppler images show that there are two prominent spot latitude belts, one near the pole and the other at lower latitudes. The differential rotation of $\mathrm{AB}$ Dor has been determined from the cross-correlation of brightness maps by Donati \& Collier Cameron (1997). According to them the relative differential rotation $\Delta \Omega / \Omega_{0} \approx 0.0046$, which is about 40 times less than in the Sun.

Its young age, strong magnetic activity and similarity to the Sun allow us to consider AB Dor as a young solar analog. Comparing its activity patterns to those of the Sun and other solar-type stars at different evolutionary stages, we can learn how the stellar magnetic activity evolves on the main sequence. For instance, the spot distribution on a young solar ana$\log$ LQ Hya, which is similar to AB Dor, comprises two active longitudes about $180^{\circ}$ apart dominating the activity of the star over 20 years (Berdyugina et al. 2002). A weak differential rotation on the stellar surface and changes of spot latitudes result in continuous migration of the active longitudes. The activity of LQ Hya is governed by three cycles: the 15 -year cycle of the total spottedness variation coupled with the differential rotation, the 7.7-year cycle of the amplitude modulation of the brightness and the 5.2-year flip-flop cycle of the alternating active longitudes. The cycle lengths are found to be in the relation of 1:2:3. The active longitudes and the three cycles imply co-existence of a non-axisymmetric and axisymmetric modes, which should be modelled with the nonlinear mean-field dynamo theory (Tuominen et al. 1999, 2002; Moss 2004).

The above results encouraged us to collect all available photometric data of $\mathrm{AB}$ Dor and analyse them with the aim to establish whether the star exhibits active longitudes and cyclic behaviour in spot patterns and overall spot activity. The observations and analysis methods are described in Sect. 2. The obtained results are presented in Sect. 3. In Sect. 4 we discuss the activity patterns of $\mathrm{AB}$ Dor and compare them with those of the Sun and more active stars and, finally, we summarise our findings in Sect. 5.

\section{Data and analysis}

\subsection{Observations}

In order to search for long-lived spot structures and activity cycles, we collected available photometric records and compiled a dataset covering 21 years from the end of 1978 to 2000 with a gap in 1998-1999. Most of the original data were in the standard Johnson UBVRI system, with the exception of data by Rucinski (1983), Kubiak (1985) and Jetsu et al. (1990), which were in the Strömgren uvby system.
Previously unpublished data from December 1990 to February 1991, November 1991 to January 1992, December 1992 to January 1993, August 1995, January 1996 and September 1996 have been observed at Molehill Astronomical Observatory (Auckland, New Zealand) using a 200mm f10 Schmidt-Cassegrain Telescope. An RCA 931b P.M.T., standard Johnson $B$ and $V$ filters and DC electronics were used. The light curves based on this data have been published by Bos (1994a).

Another set of previously unpublished data was collected in October/November 1995 and October 1996 with the $0.5 \mathrm{~m}$ telescope at the European Southern Observatory (ESO, La Silla, Chile) and in January 1999 with the 0.5 m SAAO telescope (Sutherland, South Africa). The $0.5 \mathrm{~m}$ ESO telescope was equipped with a single-channel photon-counting photometer, a thermoelectrically cooled Hamamatzu R-943/02 photomultiplier and standard ESO filters matching the $U B V(R I)_{c}$ system. The observations at SAAO were carried out with a similar photometer.

The sources of the analysed observations as well as names of comparison (C) and check (Ck) stars are given in Table 1. The unpublished data obtained at Molehill, and ESO and SAAO are given in Tables 2 and 3, respectively. The observations used are presented in Fig. 1.

The errors of the observations are typically about 0.01 or less, except of the dataset of Anders (1993), which was available only in plots of magnitude versus phase. The magnitudes were read out from the plots, and the accuracy of these data is 0.05 .

The data were divided into 48 sets, so that most of the sets had a good phase coverage over the rotational period and each set represented a light curve stable for a given time interval. The observations were phased with the ephemeris by Innis et al. (1988):

$T=2444296.575+0.51479 E$.

\subsection{Light curve inversions}

In order to determine locations of spots on the stellar surface, we performed inversions of the observed light curves into stellar images. The used approach was developed by Berdyugina et al. (2002). It is based on the Occamian inversion technique (Berdyugina 1998) which is applied to the photometric light curves in the two-temperature approximation. The model assumes that, because of low spatial resolution, a local intensity of the stellar surface contains contributions from both hot photosphere and cool spots, weighted by the fraction of the surface covered by spots - the spot filling factor. The inversion of the light curve results in a distribution of the spot filling factor over the stellar surface. Since a light curve represents one-dimensional time series the resulting stellar image contains information on the spot distribution only in one direction, in longitude, while spot extents and locations in latitude remain uncertain. However, because of the projection effect and limb darkening, light-curve inversion images show better-defined structures than the light curves themselves and, therefore, can be used to determine longitudes of spot 
Table 1. Sources of the photometric data.

\begin{tabular}{lrlll}
\hline \hline JD range & $N$ & Source & C & Ck \\
(JD 2 400 000+) & & & \\
\hline $43856-44974$ & 83 & Lloyd Evans \& & 1 & 2 \\
& & Koen (1987) & & \\
$44897-44909$ & 158 & Rucinski (1983) & 3 & 4 \\
$44982-44993$ & 74 & Rucinski (1983) & 4 & 3 \\
$45249-45266$ & 180 & Kubiak (1985) & 3 & \\
45791 & 43 & Innis et al. (1988) & 3 & 9 \\
$46019-46020$ & 18 & Innis et al. (1988) & 3 & 9 \\
$46040-46050$ & 745 & Lloyd Evans (1987) & & \\
$46043-46055$ & 614 & Jetsu et al. (1990) & 3 & 4 \\
$46096-46097$ & 40 & Innis et al. (1988) & 3 & 9 \\
$46389-46397$ & 23 & Cutispoto \& & 3 & 5,6 \\
& & Rodonò (1988) & & \\
$46445-46446$ & 14 & Innis et al. (1988) & 3 & 9 \\
$46520-46521$ & 29 & Innis et al. (1988) & 3 & 9 \\
$46703-46704$ & 100 & Innis et al. (1988) & 3 & 9 \\
$46736-46743$ & 107 & Kubiak et al. (1995) & 3 & \\
$46756-46841$ & 42 & Cutispoto (1990) & 3 & 5 \\
$46810-46811$ & 64 & Innis et al. (1988) & 3 & 9 \\
$47548-47595$ & 60 & Kürster et al. (1994) & 7 & \\
$478 x x-489 x x$ & 212 & Anders (1993) & & \\
$47870-47887$ & 57 & Cutispoto (1995) & 4 & 2 \\
$48253-50349$ & 713 & this paper, Molehill & 3 & 10 \\
$48331-48344$ & 32 & Cutispoto (1998a) & 5 & 2 \\
$48667-48680$ & 24 & Cutispoto (1998b) & 5 & 2 \\
$48987-49000$ & 14 & Cutispoto et al. (2001) 5 & 2 \\
$49304-49383$ & 304 & Bos (1994b) & 3 & 11 \\
$49311-49323$ & 34 & Unruh et al. (1995) & 5 & 2 \\
$49317-49327$ & 20 & Anders (1994) & 3 & 9 \\
$49659-49724$ & 708 & Budding et al. (1995) & 3 & 11 \\
$49681-49691$ & 34 & Cutispoto et al. (2003) 5 & 8 \\
$49970-50075$ & 287 & Bos et al. (1996) & 3 & 10,11 \\
$49992-50004$ & 38 & this paper, ESO & 5 & 2 \\
$50363-50369$ & 30 & this paper, ESO & 5 & 2 \\
$50426-50439$ & 25 & Schmitt et al. (1998) & 5 & 2 \\
$51520-51526$ & 19 & this paper, SAAO & 5 & 2 \\
\hline
\end{tabular}

Comparison $(\mathrm{C})$ and Check $(\mathrm{Ck})$ stars:

$1=\mathrm{HD} 36269 ; \quad 2=\mathrm{HD} 36316 ; \quad 3=\mathrm{HD} 37297$;

$4=\mathrm{HD} 35474 ; \quad 5=\mathrm{HD} 35230 ; \quad 6=\mathrm{HD} 38616 ;$

$7=$ HD 37292; $\quad 8=$ HD 36313; $\quad 9=$ HD 35537;

$10=$ HD 36876; $11=$ HD 37279 .

concentrations. Although this approach is less informative than the Doppler imaging technique, the analysis of long series of photometric observations allows us to recover longitudinal spot patterns and study their long-term evolution.

For AB Dor, values of the photospheric and spot temperatures were assumed to be equal to $5200 \mathrm{~K}$ and $4000 \mathrm{~K}$, respectively. The temperature of the photosphere corresponds to the effective temperature of a $\mathrm{K} 0 \mathrm{~V}$ star, while spots were assumed to be cooler by $1200 \mathrm{~K}$, which is typical for solar-type stars. Since we were interested only in spot longitudes, possible errors in the temperature values were not important. For the inversions, the stellar surface was divided into a grid of $10^{\circ} \times 10^{\circ}$, and the spot filling factor was determined for each grid pixel. The recovered stellar images and fits to the observed light curves are shown in Fig. 2.

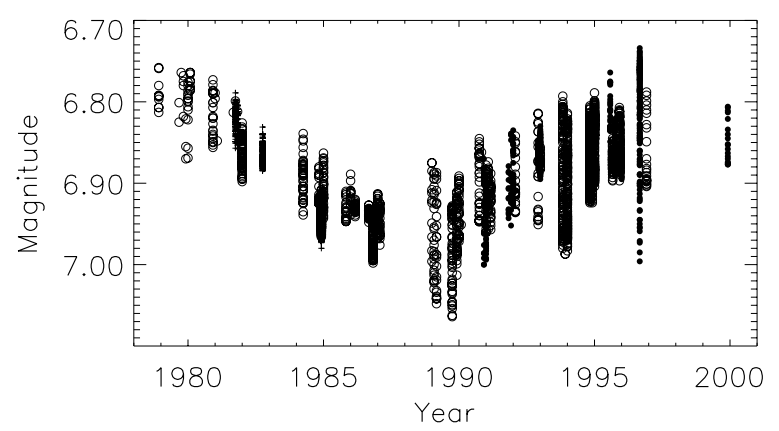

Fig. 1. The photometric data analysed in this paper. Open circles denote $V$-band photometry, + symbols denote $y$-band photometry and filled circles represent the new data.

\section{Results}

\subsection{Active longitudes}

From the images we recovered spot longitudes at the locations of the maximum spot filling factor. In many cases, two maxima were seen, which indicated two spot concentrations on the stellar surface, with one generally being larger. The time dependence of the spot phases together with error bars are shown in Fig. 3. The errors in spot phases are due to broad minima or incompleteness of the light curve. They are determined from the full width at half maxima of the spot filling factors. The photometric phases of the spots are also given in Table 4.

The two spot concentrations constitute two active longitudes about $180^{\circ}(\Delta \varphi=0.5)$ apart. As seen in Fig. 3, the active longitudes have a noticeable drift. In 1979-1983 they migrate to earlier phases, then in 1984-1994 to later phases, and after 1995 they seem to migrate again to earlier phases. Since the phases were calculated with a fixed period value, the drift indicates changes of the spot rotation period on the time scale of about 20 years.

In order to determine the spot rotation period at different times of observations, we performed a non-linear fit to the spot phases taking into account the migration of the active longitudes (shown by solid lines in Fig. 3). Two extreme values of the period are determined from the fit: $P_{\min }=0.51470$, $P_{\text {max }}=0.51487$.

\subsection{Differential rotation and a solar-type cycle}

AB Dor is known to show solar-type surface differential rotation, i.e. with more rapid rotation at lower latitudes (Donati \& Collier Cameron 1997). Therefore, different spot rotation periods at different epochs can be considered as indication that spots appear within a range of latitudes. Using the values of the spot rotation period determined from the migration of the active longitudes $P_{\min }$ and $P_{\max }$ and the differential rotation law deduced by Donati \& Collier Cameron (1997) we can determine the range of mean spot latitudes on AB Dor over the 20-year interval. This results in the minimum and maximum mean latitudes of $47^{\circ}$ and $51^{\circ}$, respectively.

The shape of the migration paths of the active longitudes implies continuous variations of the spot rotation period. In the presence of differential rotation, this suggests continuous 


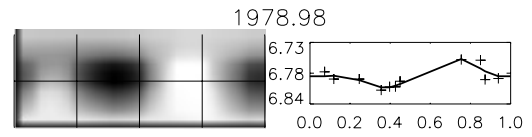

1979.91

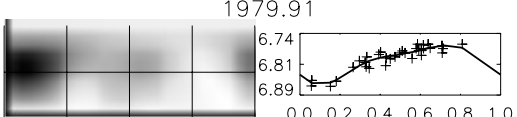

1981.03
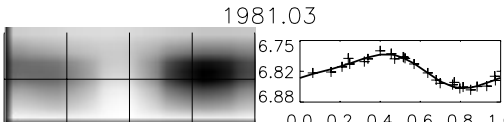

1981.80

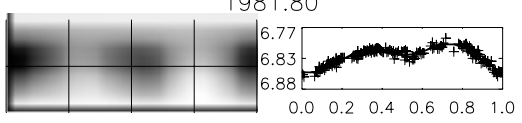

1982.04

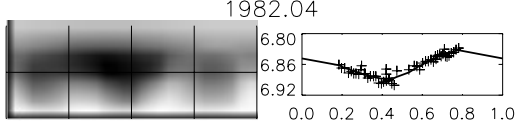

1982.50

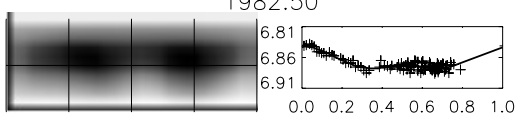

1982.80

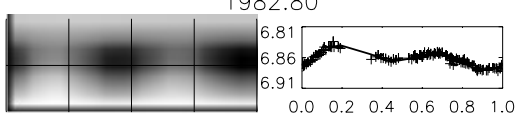

1984.25

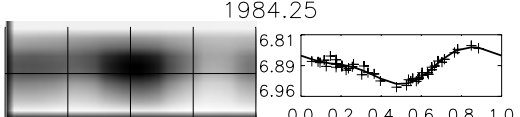

1984.87

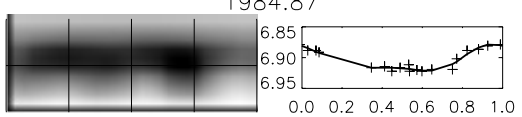

1984.93

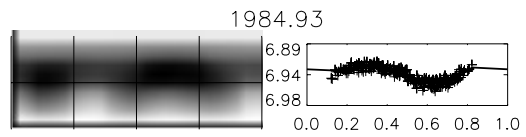

1984.94

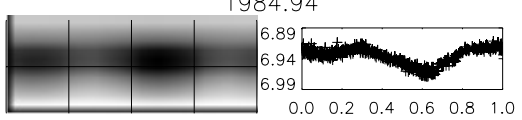

1984.95

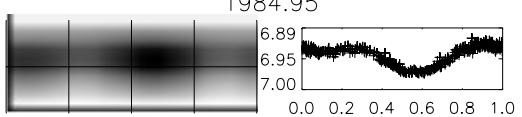

1984.97
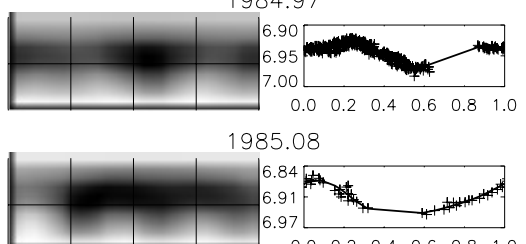

85.08

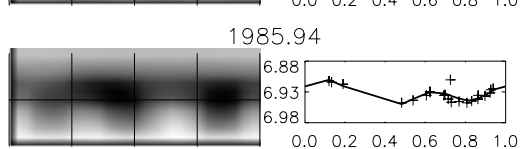

1986.04

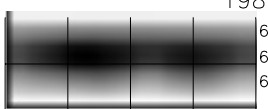

6.91

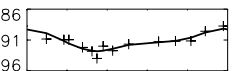

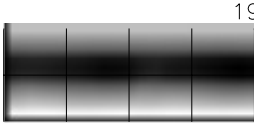

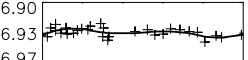

$\begin{array}{lllllll}0.0 & 0.2 & 0.4 & 0.6 & 0.8 & 1.0\end{array}$

1986.74
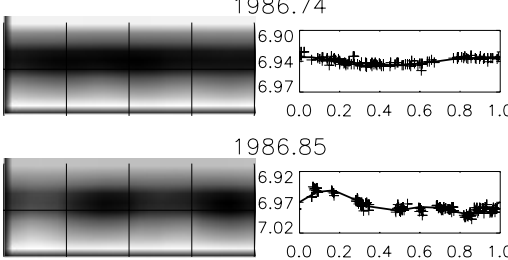

986.85
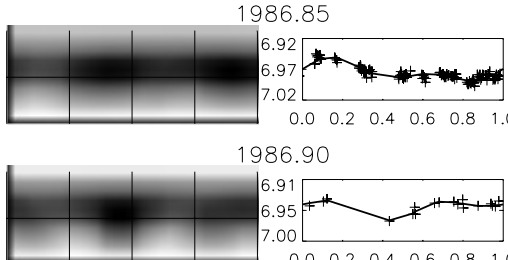

986.90

6.9
6.95
7.00

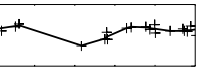

1987.04

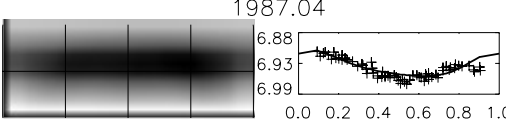

1987.11

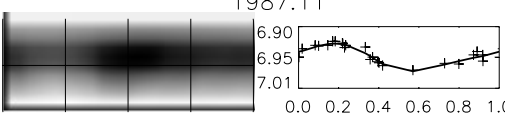

1989.12

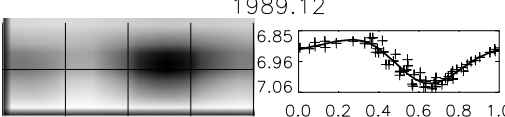

1989.79

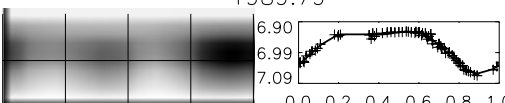

1989.96
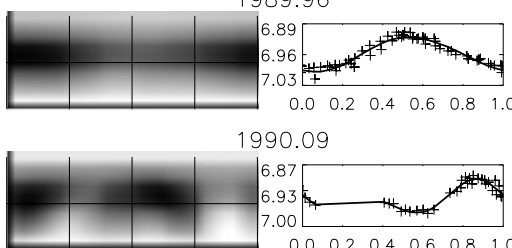

990.09

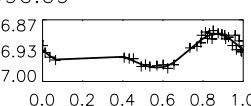

1990.84

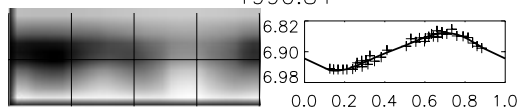

1991.00

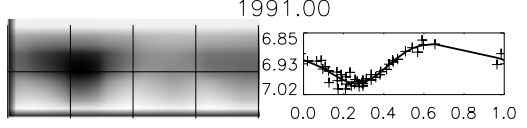

1991.04

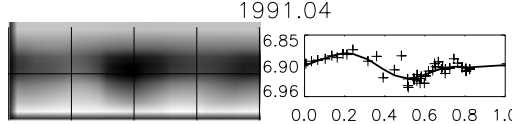

1991.12

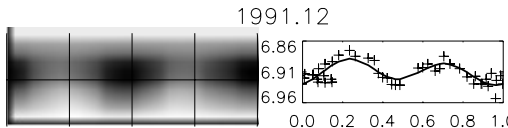

1991.22

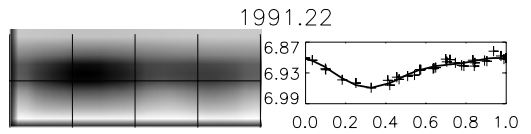

1991.96
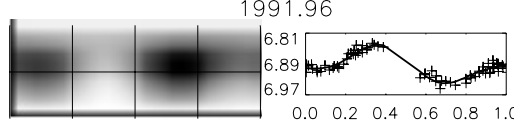

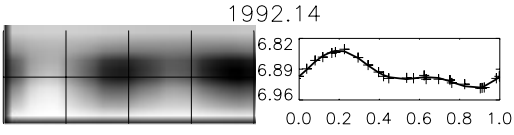

1992.96

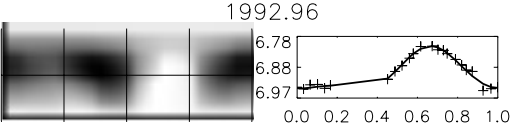

1993.01

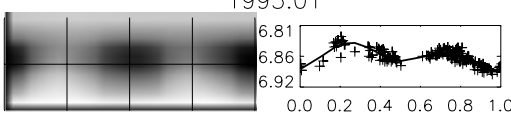

1993.07

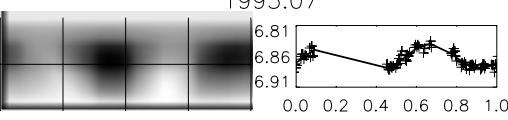

1993.89

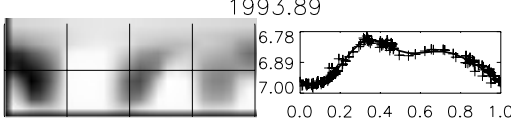

1993.92

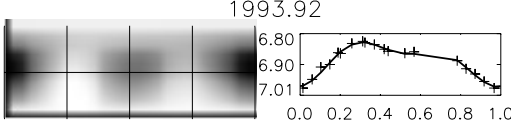

1994.05
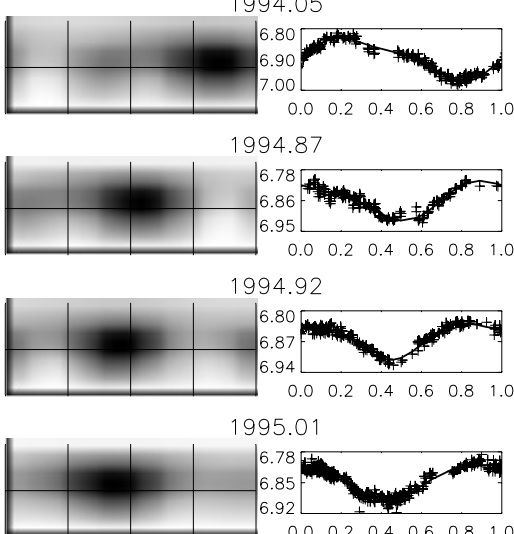

1995.68

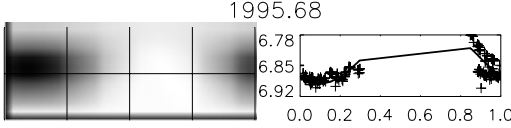

1995.77

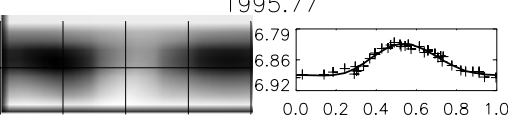

1995.99

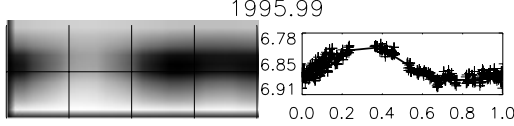

1996.72

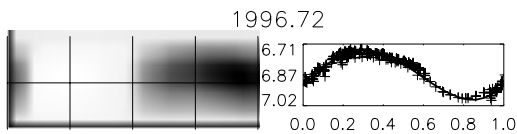

1996.97

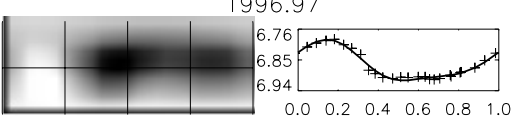

1999.94

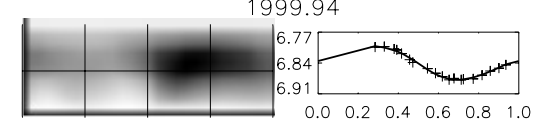

Fig. 2. The light-curve inversion results. Images are shown in a gray scale with spot filling factor being larger in darker regions. The coordinate grid in the images indicates positions of the equator and 4 longitudes separated by $90^{\circ}$. Observed and calculated light curves are presented by crosses and lines, respectively. Note the changing vertical scale in the light curve plots. 


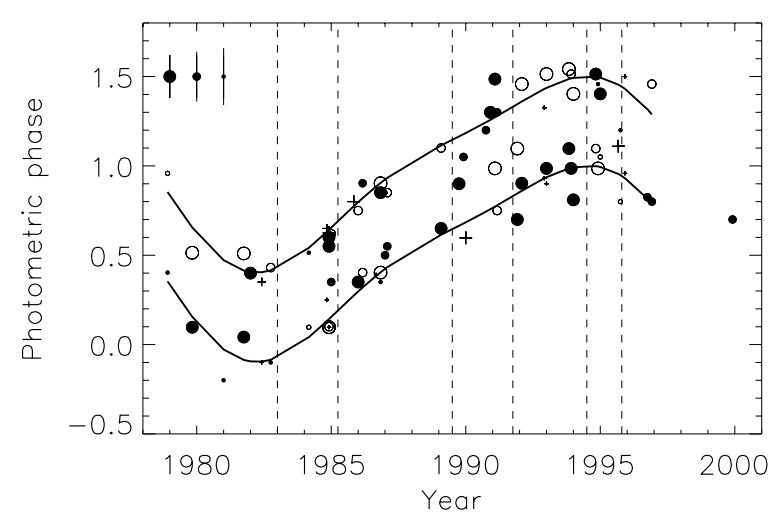

Fig. 3. Phases of the spots recovered on the surface of $A B$ Dor using the light curve inversion technique. Filled circles denote primary spots, open circles secondary spots, and + symbol is used when it is not possible to say which spot is primary one. Active longitudes are traced by solid lines. Different symbol sizes correspond to different error bars shown in the top left corner of the plot.

changes of the mean spot latitudes, with spots being on average at lower latitudes before 1983 and at higher latitudes after that. In the Sun, the mean spot latitude changes are accompanied by total sunspot area variations during the 11-year sunspot cycle. A similar behaviour is observed for $\mathrm{AB}$ Dor. As seen from Figs. 1 and 3, the overall activity level of $A B$ Dor and the spot rotation period vary on the same time scale, approximately 20 years, which suggests the presence of a sunspot-like cycle on $\mathrm{AB}$ Dor.

The length of the cycle in AB Dor can be estimated from the photometric data presented in Fig. 1. Using a second order Fourier fit to the brightness variations we find the cycle length to be about $(20-22) \pm(2-3)$ years. Fits to the maximum, mean and minimum magnitudes and the amplitude in Fig. 4 also show a cycle of about 20 years. This cycle is further confirmed by the spectral analysis of the data with the Lomb method for unevenly sampled data (Press et al. 1992). The turning point in the migration paths in 1983 may indicate the beginning of a new cycle. Because of sparse data in 1978-1985, the position of the turnover time is not well defined. In order to confirm the 20-year cycle, one needs to observe a subsequent phase turnover. It might have happened during the years 2000-2004 and, thus, the most latest photometric observations of AB Dor would be essential to confirm the cycle.

\subsection{Flip-flop cycle}

A flip-flop occurs when the dominant spot concentration switches to another active longitude. This effect was found to be periodic in RS CVn-type stars, FK Com, and the young solar analog LQ Hya (Berdyugina \& Tuominen 1998; Korhonen et al. 2002; Berdyugina et al. 2002). A series of flip-flops thus indicate a new type of stellar activity cycle which is related to active longitudes. It is therefore interesting to look for such a cycle in the activity of AB Dor.

In Fig. 3, primary spot concentrations are denoted by solid circles. One can see that they rather regularly jump from one active longitude to the other, indicating the flip-flop effect. Such

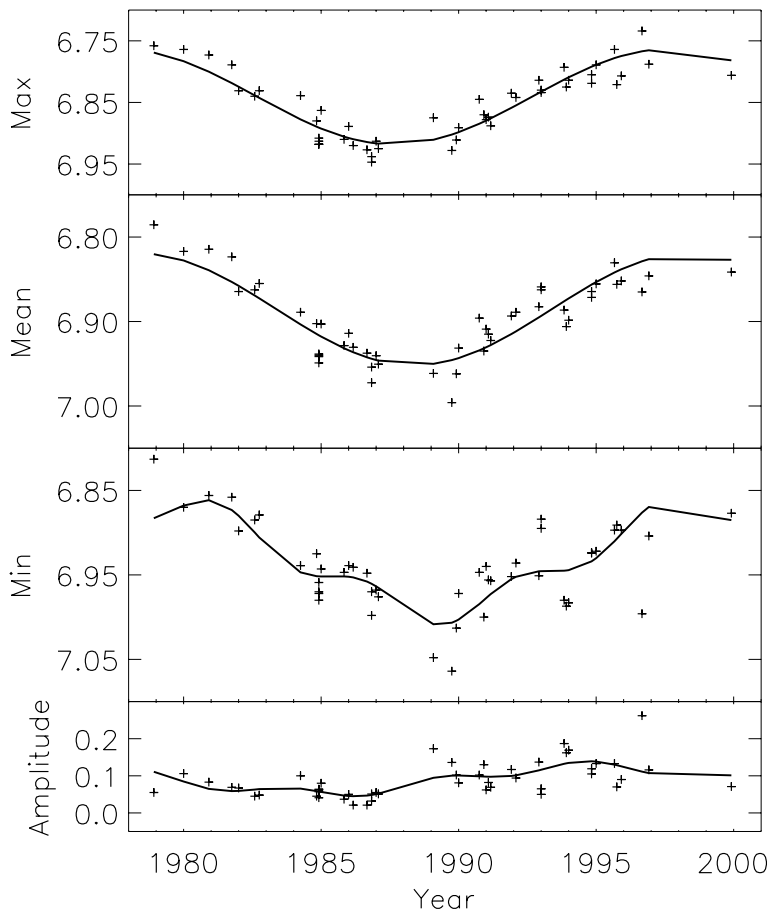

Fig. 4. Maximum, mean, minimum and peak-to-peak amplitude magnitudes measured from the individual light curves (crosses). Harmonic fits to the variations (solid lines) and the spectral analysis reveal the 20-yr and 5.5-yr cycles in the data.

moments are emphasised by vertical dashed lines. On average the flip-flops happen in about 2-3 year intervals and, thus, suggest a flip-flop cycle of about 5-6 years.

To investigate this effect statistically, we employ the procedure introduced by Berdyugina \& Usoskin (2003) for the Sun. We build a dominance vector $D$ which is equal to 0 or 1 depending on whether the major spot concentration is on the lower or upper active longitude in Fig. 3 . The discrete $D$-series is further smoothed with a window, which covers 3 steps of the dominance vector and is shifted by 1 step. The smoothed dominance series for primary spots is shown in Fig. 5, which reveals a clear periodic signal. We carried out a spectral analysis of this series using the Lomb method (Press et al. 1992). The result of the analysis is shown in Fig. 6. Two peaks are clearly revealed in the power spectrum with frequencies centred at 0.185 and $0.29 \mathrm{yr}^{-1}$ which correspond to periods of 5.4 and 3.4 years. The longer period represents the full flip-flop cycle consisting of two subsequent switches. The shorter period seemingly represents the first harmonics of the longer cycle which is revealed due to asymmetry of the flip-flop cycle. The asymmetry is expressed as an alternation of longer and shorter intervals between flip-flops: one active longitude dominates on average during 3 years, while the other during the remaining 2 years of the cycle (see Fig. 3 ).

The 5.5-yr cycle is also evident in the amplitude modulation of the brightness and affects variations of the minimum magnitude as shown in Fig. 4 and previously reported by Amado et al. (2001). Such a behaviour suggests the periodic redistribution of the spot area between the opposite longitudes and supports the reality of the flip-flop cycle. 
Table 4. Spot parameters: JD is the Julian date -2400000 in the beginning and in the end of the set, $\varphi_{1}$ and $\varphi_{2}$ are the recovered phases of the primary and secondary active regions, respectively. In the comment Col.: $1=$ incomplete light curve; $2=$ uncertain minimum; and $3=$ difficult to distinguish which spot is primary.

\begin{tabular}{|c|c|c|c|c|c|c|c|c|c|c|c|c|c|}
\hline Set & Year & JD (beg) & JD (end) & $\varphi_{1}$ & $\varphi_{2}$ & Com & Set & Year & JD (beg) & JD (end) & $\varphi_{1}$ & $\varphi_{2}$ & Com \\
\hline 1 & 1978.98 & 43856 & 43874 & 0.403 & 0.958 & 1 & 25 & 1989.96 & 47870 & 47887 & 1.150 & --- & \\
\hline 2 & 1979.91 & 44133 & 44281 & 0.097 & 0.514 & 1 & 26 & 1990.09 & 47923 & 47923 & 0.597 & --- & 1,3 \\
\hline 3 & 1981.03 & 44591 & 44638 & -0.200 & --- & 2 & 27 & 1990.84 & 48196 & 48196 & 1.200 & --- & 1,2 \\
\hline 4 & 1981.80 & 44860 & 44918 & 0.042 & 0.510 & & 28 & 1991.00 & 48253 & 48259 & 1.300 & --- & 1 \\
\hline 5 & 1982.04 & 44982 & 44989 & 0.400 & --- & 1 & 29 & 1991.04 & 48267 & 48272 & 1.486 & --- & 1 \\
\hline 6 & 1982.50 & 44993 & 45251 & -0.100 & 0.350 & 1,3 & 30 & 1991.12 & 48295 & 48303 & 1.486 & 0.986 & \\
\hline 7 & 1982.80 & 45257 & 45266 & -0.100 & 0.431 & & 31 & 1991.22 & 48331 & 48344 & 1.300 & 0.750 & \\
\hline 8 & 1984.25 & 45791 & 45791 & 0.514 & 0.097 & & 32 & 1991.96 & 48578 & 48630 & 0.700 & 1.097 & 1 \\
\hline 9 & 1984.87 & 46019 & 46020 & 0.650 & 0.250 & 1,3 & 33 & 1992.14 & 48667 & 48680 & 0.903 & 1.458 & \\
\hline 10 & 1984.93 & 46040 & 46042 & 0.625 & 0.097 & 1,3 & 34 & 1992.96 & 48972 & 48972 & 0.931 & 1.325 & 1,3 \\
\hline 11 & 1984.94 & 46043 & 46045 & 0.600 & 0.097 & & 35 & 1993.01 & 48977 & 49002 & 0.986 & 1.514 & \\
\hline 12 & 1984.95 & 46046 & 46051 & 0.550 & 0.097 & & 36 & 1993.07 & 49010 & 49016 & 0.900 & --- & 1,3 \\
\hline 13 & 1984.97 & 46052 & 46055 & 0.569 & 0.100 & 1 & 37 & 1993.89 & 49304 & 49320 & 1.097 & 1.542 & \\
\hline 14 & 1985.08 & 46096 & 46097 & 0.350 & 0.620 & 1,3 & 38 & 1993.92 & 49322 & 49327 & 0.986 & 1.514 & \\
\hline 15 & 1985.94 & 46389 & 46398 & --- & 0.800 & 1,3 & 39 & 1994.05 & 49357 & 49382 & 0.810 & 1.403 & \\
\hline 16 & 1986.04 & 46445 & 46446 & 0.350 & 0.750 & & 40 & 1994.87 & 49659 & 49682 & 1.514 & 1.097 & \\
\hline 17 & 1986.25 & 46520 & 46521 & 0.903 & 0.403 & 2,3 & 41 & 1994.92 & 49683 & 49693 & 1.458 & 0.986 & \\
\hline 18 & 1986.74 & 46695 & 46704 & 0.390 & 0.860 & 2,3 & 42 & 1995.01 & 49715 & 49724 & 1.403 & 1.050 & \\
\hline 19 & 1986.85 & 46736 & 46743 & 0.850 & 0.403 & & 43 & 1995.68 & 49956 & 49973 & 1.110 & --- & 1,3 \\
\hline 20 & 1986.90 & 46756 & 46760 & 0.350 & 0.903 & 1 & 44 & 1995.77 & 49992 & 50003 & 1.200 & 0.800 & 2,3 \\
\hline 21 & 1987.04 & 46810 & 46811 & 0.500 & 0.850 & 1,2 & 45 & 1995.99 & 50069 & 50090 & 0.958 & 1.500 & 2,3 \\
\hline 22 & 1987.11 & 46832 & 46841 & 0.550 & 0.850 & & 46 & 1996.75 & 50346 & 50369 & 0.825 & --- & \\
\hline 23 & 1989.12 & 47548 & 47595 & 0.650 & 1.100 & & 47 & 1996.97 & 50426 & & 0.800 & 1.458 & 3 \\
\hline 24 & 1989.79 & 47815 & 47815 & 0.900 & --- & 1 & 48 & 1999.94 & 51520 & 51525 & 0.700 & --- & 1 \\
\hline
\end{tabular}

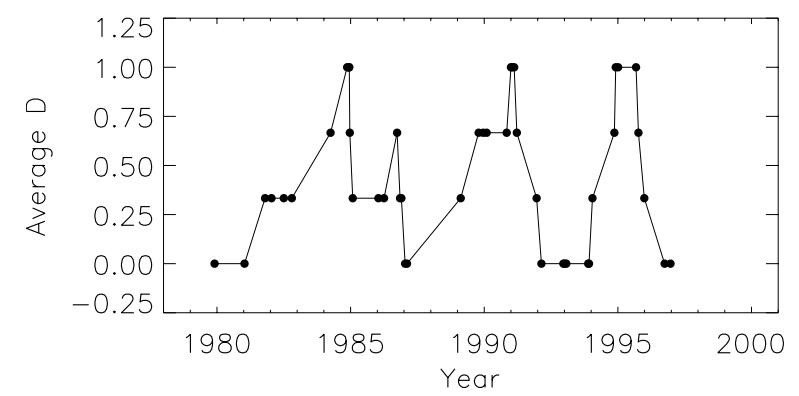

Fig. 5. The smoothed dominance for primary spots.

\section{Discussion}

Since AB Dor has been studied very extensively with the Doppler imaging technique, we can compare latitudes and longitudes of spots recovered in Doppler maps with our results for those seasons when spectroscopic and photometric observations were obtained at about the same time.

The earliest Doppler image found was obtained by Collier Cameron \& Unruh (1994, Figs. 4 and 9) in January 1992, which corresponds to the photometric set 32 (Table 4). According to our results, the major spot occupancy is expected to be at $\varphi=0.7$ and the secondary at $\varphi=1.1$ (corresponding to the longitudes $l=110^{\circ}$ and $324^{\circ}$ in the Doppler map). Unfortunately, because of the phase gap between 0.7 and 1.0 in spectroscopic observations, the primary spot is not fully recovered on the

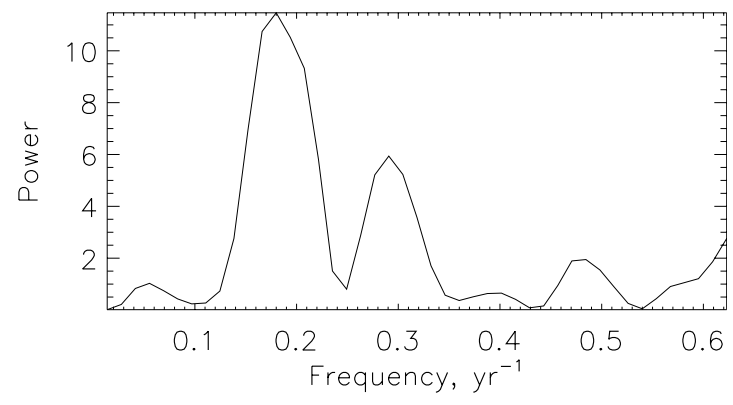

Fig. 6. Power spectrum of the smoothed dominance. The peaks centred at the frequencies of 0.185 and 0.29 correspond to the periods of 5.4 and 3.4 years.

map. However, the least spotted area corresponding to our $\varphi=0.35$ is in agreement with the longitude $l=230^{\circ}$ from the Doppler image. The average spot occupancy as a function of latitude has two peaks: at lower latitudes around $15^{\circ}$ $25^{\circ}$ and at higher latitudes around $55^{\circ}-80^{\circ}$, that lead to the mean spot latitude of about $47.5^{\circ}$, which is within our estimate of the latitude range of $47^{\circ}-51^{\circ}$. An image obtained by Collier Cameron (1995) from the same data with an improved inversion technique shows spot concentrations at phases 0.0 0.25 and $0.7-0.5$, which is in a better agreement with the set 32 .

Doppler images corresponding to the set 34 for December 1992 were published by Collier Cameron (1995, Figs. 5 and 6). Again, because of the significant phase gap in spectroscopic data, the spot at $\varphi=0.93\left(l=330^{\circ}\right)$ is not fully recovered, 
although there is an indication of it in a high latitude spot stripe. The spot at $\varphi=0.33\left(l=120^{\circ}\right)$ is found to be also concentrated at high latitudes. An absence of low-latitude spots in the Doppler map at $\varphi=0.6-0.7$ is consistent with the observed brightness maximum in set 34 .

Spectroscopic data analysed by Unruh et al. (1995) and Hussain et al. (1997) correspond to our set 37. In both papers it is concluded that spots appear at rotational phases $\varphi=0.5$ and $\varphi=0.95$, which is almost consistent with our estimates of the spot phases 0.54 and 1.1. The difference can be explained by the phase gap in the spectroscopic data around $\varphi=0.0$. The average spot occupancy as a function of latitude showed two peaks: at lower latitudes around $10^{\circ}-20^{\circ}$ and at higher latitudes around $65^{\circ}-70^{\circ}$, which leads to the mean spot latitude of about $45^{\circ}$. The spot coverage as a function of longitude also showed two maxima, corresponding to the two spot concentrations separated by about $180^{\circ}$.

The Doppler images by Collier Cameron et al. (1999, Figs. 7-9 and 11) are comparable with our set 40 . The light curve shows a broad minimum centred at $\varphi=0.5$. This is consistent with a large spotted area seen in the Doppler images spanning from $\varphi=0.5-0.6\left(l=190^{\circ}-120^{\circ}\right)$ together with a smaller spotted area at $\varphi=0.25-0.3\left(l=270^{\circ}-260^{\circ}\right)$. The spot activity peaks at latitude belts of $15^{\circ}-25^{\circ}$ and $70^{\circ}-80^{\circ}$, which again results in the spot mean latitude within the expected range.

Hussain et al. (2000) and Donati et al. (1999, 2003) presented Doppler images corresponding to our sets 45, 47 and 48, which are distinguished by broad minima. The Doppler maps suggest that the broad minima occur due to two close spot concentrations. As before, the spots appear in two latitudinal belts with the average within $45^{\circ}-50^{\circ}$.

The two active longitudes separated by about $180^{\circ}$ on AB Dor were first discussed by Innis at al. (1988) who identified two long-lived spots and determined the average period of spot rotation. Anders et al. (1992) interpreted light curves with a two-spot model and also revealed two active longitudes separated by $180^{\circ}$. A limited data set and the rapidly changing nature of the light curves did not allow the above authors to recover the migration of the active longitudes, which becomes evident on a longer time-scale.

A striking confirmation of the active longitudes on AB Dor comes from the analysis of radio emission peaks which seem to be centred on spot concentrations observed photometrically over many rotations (Lim et al. 1994). Also, a clear rotational modulation in emission of the transition layer lines C III and OIV (Ake et al. 2000) suggests that the active regions extend from the photosphere through chromosphere to corona.

\section{Summary}

In this paper, we present photometric data of the young active dwarf AB Dor and analyse them with the technique of inversion of light curves into stellar images. Such an approach reveals the longitudinal spot distribution and is good enough for the purpose of studying active longitudes and their evolution.
The following results are obtained:

- Spots on the surface of AB Dor are grouped around two active longitudes which are separated on average by $180^{\circ}$ and migrate with a variable rate due to surface differential rotation.

- The total spot area (mean brightness) varies on about 20-yr time-scale, indicating a solar-type cycle. The same cycle is seen in the migration paths of the active longitudes.

- Periodic switches of the dominant activity between the active longitudes results in a flip-flop cycle of about 5.5 years, which is also seen in the peak-to-peak amplitude of magnitude variations.

- Magnetic activity of AB Dor is similar to that observed in the other young solar analog LQ Hya, the single giant FK Com and binary components of the RS CVn-type.

Acknowledgements. We are grateful to all observers who kindly provided their data. S. P. Järvinen acknowledges support by the Deutsche Forschungsgemeinschaft, grant KO 2320/1, and S. V. Berdyugina acknowledges support by the Academy of Finland, grant 43039 .

\section{References}

Ake, T. B., Dupree, A. K., Young, P. R., et al. 2000, ApJ, 538, L87 Amado, P. J., Cutispoto, G., Lanza, A. F., \& Rodonò, M. 2001, in Proc. of the Eleventh Cambridge Workshop on Cool Stars, Stellar Systems, and the Sun, ed. R. J. García López, R. Rebolo, \& M. R. Zapatero Osorio, ASP Conf. Ser., 223, CD-895

Anders, G. J. 1993, Ph.D. Thesis, Monash University, Australia Anders, G. J. 1994, IBVS, 3985

Anders, G. J., Coates, D. W., \& Thompson, K. 1992, PASA, 10, 33

Berdyugina, S. V. 1998, A\&A, 338, 97

Berdyugina, S. V., \& Tuominen, I. 1998, A\&A, 336, L25

Berdyugina, S. V., \& Usoskin, I. G. 2003, A\&A, 405, 1121

Berdyugina, S. V., Pelt, J., \& Tuominen, I. 2002, A\&A, 394, 505

Bidelman, W. P., \& MacConnell, D. J. 1973, AJ, 78, 687

Bos, M. 1994a, ExA, 5, 13B

Bos, M. 1994b, IBVS, 4111

Bos, M., Hudson, G., Hudson, R., \& Budding, E. 1996, IBVS, 4330

Budding, E., Hudson, G., \& Hudson, R. 1995, IBVS, 4203

Collier Cameron, A. 1995, MNRAS, 275, 534

Collier Cameron, A., Bedford, D. K., Rucinski, S. M., et al. 1988, MNRAS, 231, 131

Collier Cameron, A., \& Unruh, Y. C. 1994, MNRAS, 269, 814

Collier Cameron, A., Walter, F. M., Vilhu, O., et al. 1999, MNRAS, 308,493

Cutispoto, G. 1990, A\&AS, 84, 397

Cutispoto, G. 1995, A\&AS, 111, 507

Cutispoto, G. 1998a, A\&AS, 127, 207

Cutispoto, G. 1998b, A\&AS, 131, 321

Cutispoto, G., \& Rodonò, M. 1988, IBVS, 3232

Cutispoto, G., Messina, S., \& Rodonò, M. 2001, A\&A, 367, 910

Cutispoto, G., Messina, S., \& Rodonò, M. 2003, A\&A, 400, 659

Donati, J.-F., \& Collier Cameron, A. 1997, MNRAS, 291, 1

Donati, J.-F., Collier Cameron, A., Hussain, G. A. J., \& Semel, M. 1999, MNRAS, 302, 437

Donati, J.-F., Collier Cameron, A., Semel, M., et al. 2003, MNRAS, 345,1145

Hussain, G. A. J., Unruh, Y. C., \& Collier Cameron, A. 1997, MNRAS, 288, 343 
Hussain, G. A. J., Donati, J.-F., Collier Cameron, A., \& Barnes, J. R. 2000, MNRAS, 318, 961

Hussain, G. A. J., van Ballegooijen, A. A., Jardine, M., \& Collier Cameron, A. 2002, ApJ, 575, 1078

Innis, J. L., Thompson, K., \& Coates, D. W. 1986, MNRAS, 223, 183

Innis, J. L., Thompson, K., Coates, D. W., \& Lloyd Evans, T. 1988, MNRAS, 235, 1411

Jetsu, L., Vilhu, O., \& la Dous, C. 1990, A\&AS, 85, 1127

Korhonen, H., Berdyugina, S. V., \& Tuominen, I. 2002, A\&A, 390, 179

Kubiak, M. 1985, Acta Astron., 35, 369

Kubiak, M., Stepień, K., Kiraga, M., \& Jahn, K. 1995, Acta Astron., 45,279

Kürster, M., Schmitt, J. H. M. M., \& Cutispoto, G. 1994, A\&A, 289, 899

Lim, J., White, S. M., Nelson, G. J., \& Benz, A. O. 1994, ApJ, 430, 332

Lloyd Evans, T. 1987, SAAOC, 11, 73

Lloyd Evans, T., \& Koen, M. C. J. 1987, SAAOC, 11, 21

Micela, G., Fatava, F., \& Sciortino, S. 1997, A\&A, 326, 221
Moss, D. 2004, MNRAS, 352, L17

Pakull, M. W. 1981, A\&A 104, 33

Press, W. H., Teukolsky, S. A., Vetterling, W. T., \& Flannery, B. P. 1992 in Numerical Recipes in FORTRAN - The Art of Scientific Computing, Second edition, 569 (New York, USA: Cambridge University Press)

Rucinski, S. M. 1982, IBVS, 2203

Rucinski, S. M. 1983, A\&AS, 52, 281

Rucinski, S. M. 1985, MNRAS, 215, 591

Slee, O. B., Nelson, G. J., Innis, J. L., et al. 1986, PASA, 6, 312

Schmitt, J. H. M. M., Cutispoto, G., \& Krautter, J. 1998, ApJ, 500, 25

Tuominen, I., Berdyugina, S. V., Korpi, M. J., et al. 1999, in Stellar Dynamos: Nonlinearity and Chaotic Flows, ed. M. Núnez, \& A. Ferriz-Mas, ASP Conf. Ser., 178, 195

Tuominen, I., Berdyugina, S. V., \& Korpi, M. J. 2002, AN, 323, 367

Unruh, Y. C., Collier Cameron, A., \& Cutispoto, G. 1995, MNRAS, 277,1145

Vilhu, O., Gustafsson, B., \& Edvardsson, B. 1987, ApJ, 320, 850

Zuckerman, B., Song, I., \& Bessell, M. S. 2004, ApJ, 613, 65 